\title{
CONTROL DE LA OFERTA DE NARANJA EN MÉXICO COMO MECANISMO PARA CONTROLAR VOLATILIDAD DE PRECIOS
}

\author{
CONTROL OF ORANGE SUPPLY IN MEXICO AS A MECHANISM \\ TO CONTROL PRICE VOLATILITY
}

\section{Alejandro Martínez-Jiménez', José A. García-Salazar*, Gabino García-de los Santos², Gustavo Ramírez-Valverde ${ }^{3}$, José S. Mora-Flores' y Jaime A. Matus-Gardea ${ }^{1}$}

\begin{abstract}
'Colegio de Postgraduados, Programa de Economía, Montecillo, Texcoco, Estado de México, México. ${ }^{2}$ Colegio de Postgraduados Programa de Producción de Semillas, Montecillo, Texcoco, Estado de México, México. ${ }^{3}$ Colegio de Postgraduados, Programa de Estadística, Montecillo, Texcoco, Estado de México, México.
\end{abstract}

*Autor de correspondencia (jsalazar@colpos.mx)

\section{RESUMEN}

Los productores de naranja (Citrus sinensis L.) en México se enfrentan al problema de bajos precios en el periodo de enero a mayo, lo que afecta su nivel de ganancia. Para determinar una medida de control de la oferta que podría disminuir la extrema variabilidad de precios se formuló un modelo de equilibrio espacial e intertemporal del mercado de la naranja en el periodo 2014-2016 bajo tres escenarios: 1) almacenar la producción, 2) desfasar la producción y 3) enviar la producción a la industria. Los resultados indican que la instrumentación de políticas de almacenamiento y la compra de la naranja por parte de la industria en los meses de mayor producción disminuiría en 81 y 115 millones de pesos mexicanos, respectivamente, las ganancias del productor, mientras que el desfase de la producción aumentaría las ganancias en 32 millones de pesos mexicanos. Una reducción en la oferta de $29 \%$ en marzo y aumento en enero, febrero y abril en $0.2,16$ y $28 \%$, respectivamente, como política de desfase de producción, aumentaría la ganancia unitaria mensual promedio en 11.75 pesos mexicanos por tonelada. Se recomienda promover la organización de productores para lograr el ordenamiento del mercado a través de la planeación de la producción en el tiempo para alcanzar mayores ingresos y una estabilidad en el precio de la fruta.

Palabras clave: Citrus sinensis, ganancia, modelo de equilibrio, organización de productores, planeación, variabilidad de precios.

\section{SUMMARY}

Orange (Citrus sinensis L.) producers in Mexico face the problem of low prices, which affects their profit level, from January to May. In order to determine a supply control measure that could reduce the extreme price variability, a spatial and inter-temporal equilibrium model of the orange market was formulated in the 2014-2016 period under three scenarios: 1) storing production, 2) desynchronizing production and 3) shipping production to industry. Results indicate that the implementation of storage policies and the purchase of oranges by the industry in the months of most massive production would decrease the producer profits by 81 and 115 million Mexican pesos, respectively. In comparison, desynchronization of production would increase profits by 32 million Mexican pesos. A $29 \%$ reduction in supply in March and increases by $0.2,16$, and $28 \%$ in January, February and April, respectively, as a policy of production shift, would increase the average monthly profit by 11.75 Mexican pesos per ton. Producer organization is recommended to achieve the ordering of the market through the planning of production over time to obtain higher incomes and stability in the price of the fruit..

Index words: Citrus sinensis, equilibrium model, planning, price variability, producer organization, profit.

\section{INTRODUCCIÓN}

La naranja (Citrus sinensis L.) es la fruta más importante en el sector agrícola de México, con una superficie de 339 mil hectáreas y una producción de 4738 mil toneladas en el año 2018 (SIAP, 2020). Al igual que la mayoría de las frutas y hortalizas producidas en México, la naranja presenta el problema de bajos precios en los meses de producción alta; en el periodo 2014-2016, el precio nacional promedio al mayoreo de la naranja en los meses de enero, febrero, marzo y abril fue inferior en 26.2, 27.4, 21.3 y $9.1 \%$, respectivamente, en relación con el precio nacional anual promedio que se ubicó en \$ 6106 pesos mexicanos por tonelada. Los bajos precios de la naranja en esos meses son consecuencia de los excesos temporales de oferta, por lo que hay una relación inversa entre la producción y los precios al mayoreo.

La producción de naranja está concentrada principalmente en los estados de Veracruz y Tamaulipas, los cuales generan el $68.2 \%$ de la oferta nacional; le siguen otros estados como Nuevo León con $7.0 \%$, San Luis Potosí con $6.9 \%$, Puebla con $4.6 \%$, Sonora con $3.2 \%$ y Yucatán con $2.9 \%$. Así mismo, la producción de este cítrico está concentrada en el tiempo; el 11.6, 18.0 y $11.7 \%$ de la oferta nacional se obtiene en los meses de febrero, marzo y abril respectivamente, mientras que en julio, agosto y septiembre se obtiene sólo el 2.4, 2.6 y 1.9 $\%$, respectivamente, de la producción nacional (SIAP, 2020). Esta característica determina que el comportamiento de la producción nacional tenga un fuerte impacto sobre los precios en los principales mercados consumidores, resultando en una relación negativa entre la cantidad nacional producida de naranja y los precios al mayoreo en los mercados nacionales, tal como lo indica la regresión simple de estas dos variables a partir de información del SNIIM (2016) y del SIAP (2016), la cual señala que si la 
producción nacional de naranja (variable independiente en el modelo) aumentara en 1000 t entonces el precio al mayoreo en la Ciudad de México (variable dependiente) disminuiría en -3.16 pesos por tonelada; de manera similar, si la producción de naranja aumentara en 1000 t, el precio al mayoreo en las centrales de abasto de Monterrey y Guadalajara disminuiría en -3.70 y -2.93 pesos mexicanos por tonelada, respectivamente.

Debido a que el exceso de oferta es la principal causa de la disminución del precio de la naranja, entonces se podrían aplicar políticas de administración de la oferta para solucionar el problema. Si se considera que el comercio exterior de naranja no es muy importante en México (SIAP, 2019); entonces, una eficiente administración de la oferta podría estabilizar los precios.

La volatilidad de los precios es un fenómeno que tiene efectos negativos sobre el ingreso del productor (RamosSandoval et al., 2018) y pone en riesgo la seguridad alimentaria de un país; además, el nivel de dicha volatilidad en los mercados de productos básicos también perjudica las expectativas de crecimiento económico y reducción de la pobreza de países en desarrollo (HLPE, 2011); por ello, algunos países han adoptado instrumentos de políticas para abordar la volatilidad de los precios.

En la India, como en otros países de Asia, se han creado programas de redes de seguridad a través de políticas de precios agrícolas que implican adquisiciones, almacenamiento y distribución, lo cual incluye un programa de logística y de almacenamiento de productos agrícolas para el control de la volatilidad de precios (HLPE, 2011). En los EUA el instrumento principal para ordenar los mercados de frutas, hortalizas y cultivos especiales han sido las políticas de administración de la oferta a través de las órdenes de mercadeo, tanto federales como estatales, para ayudar a los productores a estabilizar los mercados y aumentar los precios para incrementar las ganancias del productor, estos instrumentos tienen sus orígenes en el Acuerdo de Comercialización Agrícola de 1937 y en el marco legal permanente basado en la Ley de Ordenación de 1938 y en la Ley de Agricultura de 1949 que regían el sostenimiento de los precios de los productos básicos. El Departamento de Agricultura de EUA reporta la aplicación de órdenes de mercado para almendras (Prunus dulcis), chabacanos (Prunus armeniaca), aguacates (Persea americana), cerezas (Prunus cerasus), agrios (Oxalis pescaprae), arándanos (Vaccinium myrtillus), dátiles (Phoenix dactylifera), uvas (Vitis vinifera), avellanas (Corylus avellana), kiwis (Actinidia deliciosa), olivos (Olea europaea), cebollas (Allium cepa), peras (Pyrus communis), nueces pecanas (Carya illinoinensis), pistachos (Pistacia vera), ciruelas (Prunus domestica), papas (Solanum tuberosum), pasas, aceite de hierbabuena (Mentha spicata), tomates (Solanum lycopersicum) y nueces (Juglans regia) (AMS/ USDA, 2019).

Algunas órdenes de mercadeo especifican el grado, el tamaño, la calidad, la madurez y los requerimientos de empaque, mientras que otras regulan la cantidad máxima de producto que se puede colocar en el mercado a través del control de los volúmenes; otras autorizan actividades de investigación y propaganda; además, las órdenes de mercadeo tienen como propósito mantener la alta calidad de las frutas y hortalizas comercializadas, normalizar envases, regular el flujo del producto al mercado, establecer reservas de productos almacenables y autorizar investigación y desarrollo, así como publicidad.

Para el caso de Brasil, las asociaciones de productores de naranja y sus derivados realizan inventarios de árboles y superficies cosechadas con el fin de hacer ajustes en las cadenas productivas agrícolas cuando hay exceso de oferta, crecimiento de existencias y, en consecuencia, volatilidad en precios (De Barros et al., 2016).

En México, el problema de excesos de oferta y caída estacional de los precios para productos de exportación era atendido por la desaparecida Unión Nacional de Productores de Hortalizas (UNPH). Los principales instrumentos para ordenar las cantidades enviadas a los mercados externos de frutas y hortalizas fueron la programación de siembras y el control sobre los volúmenes exportados. Una vez que se determinaban las expectativas de cada mercado, los delegados de la UNPH y productores determinaban la superficie a sembrar por estado, lo que evitaba la saturación del mercado (Espinoza et al., 2005).

La organización anterior funcionó hasta principios de los 1990s (Macías, 2000) y posteriormente cambió a la Confederación Nacional de Productores de Hortalizas en 1989. Desde la desaparición de la UNPH, los intentos de organizar a los productores de hortalizas, con la finalidad de ordenar el mercado, se han dado de manera aislada; por ejemplo, en la Comarca Lagunera existen antecedentes de administración de la oferta a través de la siembra programada en cultivos como el melón (Cucumis melo) y la sandía (Citrullus lanatus); sin embargo, aunque en algunos municipios como Tlahualilo los calendarios de riego han sido usados para programar las épocas de siembra (Espinoza, 2003; Com. Pers.)', se requieren mayores esfuerzos en la programación de siembra, sobre

\footnotetext{
'Espinoza A. J. J. (2003) Programación de siembras de melón en la Comarca Lagunera. El Siglo de Torreón, edición 17 de agosto de 2003. Torreón, Coahuila, México. https://www.elsiglodetorreon. com.mx/noticia/45056.programacion-de-siembras-demelon-en-la-comarca-lagunera-agropecuaria.html (Enero 2020).
} 
todo en los municipios con mayor producción. Espinoza et al. (2005) reportaron que los productores de melón de la Comarca Lagunera no están organizados y que actúan de manera individual, lo cual reduce su poder de negociación con los agentes intermediarios que acuden a la región a comprar melón y sandía.

Debido a que la evidencia empírica sugiere que el control de volúmenes de producción aumenta los precios al productor (Powers, 1990), éstos pueden ser usados para estabilizar los precios de la naranja. El control de volúmenes incluye el control de flujos de mercado, la distribución de mercados, las reservas y la asignación de mercados. Estas regulaciones establecen un techo sobre la cantidad máxima de producción que pueden ingresar a ciertos mercados durante una estación, o en un periodo dentro de la estación.

Si las órdenes de mercadeo no llegaran a legislarse en un futuro próximo en México, se podrían aplicar medidas de control de la oferta en la región para evitar la caída estacional de los precios. La contracción de la oferta en los meses de mayor producción y el control del flujo de producto destinado al mercado, así como la planeación de la producción en el tiempo, serían algunas medidas que podrían implementarse para solucionar el problema. Cada medida tendría efectos diferentes sobre la ganancia del productor y cada una enfrentaría obstáculos para implementarse en la práctica.

Los bajos precios al mayoreo son transmitidos al productor, lo que disminuye sus ingresos. Puesto que menores niveles de ganancia no son deseables para el sector naranjero de México, este estudio tuvo por objetivo determinar la medida más efectiva de control de la oferta para ordenar el mercado nacional de la naranja.

\section{MATERIALES Y MÉTODOS}

\section{Modelo de programación}

Se usó un modelo de programación que incorpora las dimensiones espaciales y temporales de la producción y el consumo de naranja en México, cuya formulación se basó en la teoría de la empresa. La función objetivo maximiza la ganancia total de los productores e intermediarios. Tras asumir $i(i=7,2 \ldots l=27)$ como estados productores de naranja, $j(j=1,2 \ldots J=32)$ como mercados consumidores de naranja en fresco, $d(d=1,2 \ldots D=7)$ como estados consumidores de naranja para la industria, $m(m=1)$ como puertos de entrada de las importaciones de naranja, e $(e=$ 1) como puertos de salida de las exportaciones de naranja y $t(t=7,2 \ldots T=12)$ como meses del año, el modelo de programación se planteó en los siguientes términos:

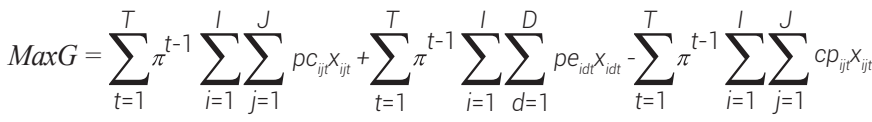

$$
\begin{aligned}
& -\sum_{t=1}^{T} \pi^{t-1} \sum_{i=1}^{l} \sum_{d=1}^{D} c p_{i d t} x_{i d t}+\sum_{t=1}^{T} \pi^{t-1} \sum_{e=1}^{E} p e_{e t} x_{e t} \sum_{t=1}^{T} \pi^{t-1} \sum_{m=1}^{M} p i_{m i} x_{m t}
\end{aligned}
$$

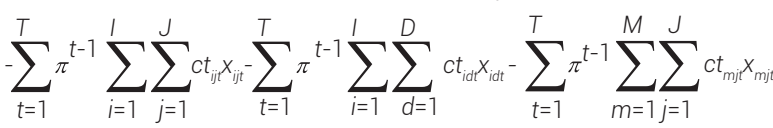

$$
\begin{aligned}
& -\sum_{t=1}^{T} \pi^{t-1} \sum_{i=1}^{1} \sum_{e=1}^{E} c t_{i e t} x_{i e t}-\sum_{t=1}^{T} \pi^{t-1} \sum_{i=1}^{1} c a_{t i t+1} x_{t i t+1}
\end{aligned}
$$

sujeto a:

$$
\begin{gathered}
\sum_{i=1}^{1} x_{j i t}+\sum_{m=1}^{M} x_{m j t} \geq y_{j t} \\
\sum_{i=1}^{1} x_{i d t} \geq y_{d t} \\
\sum_{j=1}^{J} x_{i j t}+\sum_{d=1}^{D} x_{i j t}+\sum_{e=1}^{E} x_{i e t}+\delta * x_{i t t} \leq x_{i t}+x_{i t-1, t}-x_{i t, t+1}
\end{gathered}
$$

$$
\sum_{j=1}^{J} x_{m j t} \geq x_{m t}
$$

$$
\sum_{i=1}^{1} x_{\text {iet }} \geq x_{\text {et }}
$$

$$
y_{j t^{\prime}} y_{d t^{\prime}} x_{i t^{\prime}} x_{i j t t^{\prime}} x_{i d t^{\prime}} \cdots i_{i t, t+1} \geq 0
$$

donde: para el mes $t, \pi^{t-1}=\left(1 / 1+i_{t}\right)^{t-1}$ es el factor de descuento con $i_{t}$ igual a la tasa de inflación; $p c_{i j t}$ es el precio al mayoreo de la naranja en el mercado $j$ proveniente del origen $i ; x_{i j t}$ es la cantidad de naranja enviada de la región $i$ al mercado $j$; $p e_{i d t}$ es precio de entrada a la industria de jugo de naranja en la región $d$ proveniente del origen $i ; x_{i d t}$ es la cantidad de naranja enviada de la región $i$ a la región industrial $d$; $c p_{i j t}$ es el costo de producción de naranja en i que será consumido en el mercado $j ; c p_{\text {idt }}$ es el costo de producción 
de la naranja en i que será enviado a la industria d; pi es el precio internacional de las exportaciones que saldrán por el puerto $e ; x_{e t}$ es la cantidad exportada por el puerto $e ; p i_{m t}$ es el precio internacional de las importaciones de naranja que se internarán por el puerto $m ; x_{m t}$ es la cantidad importada de naranja por el puerto $m ; c t_{i j t}$ es el costo de transporte de enviar naranja de $i$ a $j ; x_{i j t}$ es la cantidad enviada de naranja de $i$ a j; $c t_{\text {idt }}$ es el costo de transporte de enviar naranja de $i$ a $d$; $x_{i d t}$ es la cantidad enviada de naranja de $i$ a $d ; c t_{m i t}$ es el costo de transporte de enviar naranja de $m$ a $j ; x_{m j t}$ es la cantidad enviada de naranja de $m$ a $j ; c t_{i e t}$ es el costo de transporte de enviar naranja de $i$ a $e ; x_{i e t}$ es la cantidad enviada de naranja de i a e; $c a_{i t+t+1}$ es el costo de almacenamiento de naranja en la región $i$ del mes $t$ al mes $t+7 ; x_{i t, t+1}$ es la cantidad de naranja almacenada en $i$ de $t$ a $t+7$, y $\delta$ es el porcentaje de la producción de naranja que se pierde en mermas durante el proceso de comercialización.

La función objetivo estuvo sujeta a restricciones de balance de oferta y demanda. La restricción 2 indica la forma en que se abastece el consumo del mercado $j$; la restricción 3 indica cómo se abastece el consumo de las industrias; la restricción 4 indica la forma en que se distribuye la producción de cada región $i$; las restricciones 5 y 6 establecen un límite a las importaciones y exportaciones de naranja, que son muy pequeñas; la restricción 7 establece las condiciones de no negatividad del modelo.

Para incorporar el efecto que la cantidad producida (o disponibilidad del producto) tiene sobre el precio al mayoreo de la naranja, este último se calculó como sigue:

$$
p c_{i j t}=\theta_{i j t}+\eta_{i j t}\left[\sum_{i=1}^{1} x_{i t}+\sum_{i=1}^{1} \sum_{t=1}^{T} x_{i t-1, t}-\sum_{i=1}^{1} \sum_{t=1}^{T} x_{i t, t+1}\right]
$$

donde, $\theta_{i j t}$ y $\eta_{i j t}$ son la ordenada y coeficiente de la función que relaciona el precio de la naranja en los mercados $j$ con la producción total obtenida en el país en el mes t.

Debido a que el precio al productor $\left(\mathrm{pp}_{\mathrm{ijt}}\right)$ también es afectado por la oferta temporal de naranja, éste fue calculado restando al precio al mayoreo el margen de comercialización $\left(m_{i j t}\right)$ que existe entre los precios al productor y mayoreo:

$$
p p_{i j t}=p c_{i j t}-m_{i j t}
$$

Las ganancias del intermediario y del productor están dadas por las ecuaciones 10 y 11 :

$$
\begin{aligned}
& G_{i n}=\sum_{t=1}^{T} \pi^{t=1} \sum_{i=1}^{l} \sum_{j=1}^{J} p c_{i j t} x_{i j t}-\sum_{t=1}^{T} \pi^{t=1} \sum_{i=1}^{l} \sum_{j=1}^{J} p p_{i j t} x_{i j t}-\sum_{t=1}^{T} \pi^{t=1} \sum_{i=1}^{l} \sum_{j=1}^{J} c t_{i j t} x_{i j t} \\
& G_{p r o}=\sum_{t=1}^{T} \pi^{t=1} \sum_{i=1}^{l} \sum_{j=1}^{J} p p_{i j t} x_{i j t}-\sum_{t=1}^{T} \pi^{t=1} \sum_{i=1}^{l} \sum_{j=1}^{J} c p_{i j t} x_{i j t}-\sum_{t=1}^{T} \pi^{t=1} \sum_{i=1}^{l} c a_{i t, t+1} x_{i t, t+7}
\end{aligned}
$$

La ganancia del intermediario es igual al ingreso derivado por la venta de la naranja menos los costos de comprar la naranja al productor, menos los costos de transporte; a su vez, la ganancia del productor es igual al ingreso total por la venta del producto menos los costos de producción y almacenamiento. El costo de almacenamiento que se ingresó al modelo es el costo por la renta por mes de cuartos fríos especializados para frutos perecederos, obtenidos mediante cotizaciones. Es conveniente mencionar que, por ser un producto perecedero, la naranja para su consumo en fresco usualmente se mueve de las zonas productoras a los mercados consumidores; si la naranja es almacenada por más de tres meses empieza a disminuir su calidad (Pérez-Aparicio et al., 2007), lo que significa que no puede almacenarse por largos periodos de tiempo, como sería el caso de los granos. Los costos de almacenamiento se introducen al modelo para plantear un escenario hipotético y analizar lo que pasaría con la ganancia de los productores si éstos son capaces de almacenar el producto.

Para alcanzar el objetivo se plantearon cuatro soluciones del modelo. La primera consideró la situación observada en el año promedio 2014-2016, donde no existe almacenamiento; la segunda contempló la aplicación de un programa de almacenamiento y el control del flujo del producto al mercado, bajo el supuesto de un consumo de 290 mil toneladas en los meses de marzo, abril y mayo, y de 188 mil toneladas en los meses de junio, julio y agosto. El procedimiento para distribuir el consumo por mes en el segundo escenario fue el siguiente: a) se calculó el consumo semestral de marzo a agosto; b) se supuso que $20 \%$ del consumo semestral se realizó en cada uno de los tres primeros meses del periodo (marzo, abril y mayo) y $13.3 \%$ en cada uno de los siguientes tres meses (junio, julio y agosto); c) dicha distribución consideró la disponibilidad total de naranja en los seis meses. La tercera solución consideró que la producción de marzo disminuye 29 \% y ésta aumenta en los meses de enero, febrero y abril en 0.2 , 16 y $28 \%$, respectivamente. La cuarta solución supuso que la industria de naranja compra el 20 \% de la producción durante los meses de mayor producción (diciembre a mayo). 


\section{Recopilación de información}

La producción de naranja por municipio y mes se obtuvo mediante la información reportada por el SIAP (2020). El consumo de naranja en fresco e industrial se obtuvo de la siguiente manera: para calcular el volumen de consumo nacional en fresco se le restó al consumo nacional aparente (producción + importaciones - exportaciones) el volumen destinado para la industria y el volumen de pérdidas que se presentan durante el manejo postcosecha (escaldado, índice de podredumbre y magulladuras) y el consumo industrial se tomó del volumen promedio anual procesado de naranja en México reportado por el USDA (2017), este volumen se distribuyó a los diferentes estados donde se encuentran instaladas las industrias, de acuerdo con su capacidad de procesamiento.

Los precios al mayoreo en las centrales de abasto provinieron del SNIIM (2016). Los costos de producción se obtuvieron de FIRA (2017), INIFAP (2018) y entrevistas directas a productores de naranja de las zonas productoras y el precio al productor provino del SIAP (2016).

Para obtener los costos de transporte por camión de las regiones productoras a las regiones consumidoras de naranja se estimó una función donde los costos de transporte $(c t)$ constituyeron la variable dependiente y la distancia $(\mathrm{km})$ la variable independiente; con el cálculo de esta función se obtuvo una ordenada al origen y un coeficiente asociado con la distancia, los cuales se multiplicaron con una matriz de distancias entre regiones productoras y consumidoras. La información para la solución de la función provino de empresas dedicadas al transporte de frutas y perecederos de rutas representativas. La matriz de distancias se creó con información de la SCT (2017), la cual incluyó 27 puntos de origen que son los siguientes: Álamo Temapache (Ver.), Castillo de Teayo (Ver.), Martínez de la Torre (Ver.), Papantla (Ver.), Tihuatlán (Ver.), Padilla (Tam.), Xicotencatl (Tam.), Mexicali (BC), Comondú (BCS), Campeche (Cam.), Chilón (Chis.), Villa de Álvarez (Col.), San Luis Acatlán (Gro.), San Felipe Orizatlán (Hgo.), La Huerta (Jal.), Mújica (Mich.), Jojutla (Mor.), General Terán (NL), Matías Romero (Oax.), Hueytamalco (Pue.), Arroyo Seco (Qro.), José María Morelos (QRoo.), Cd. Fernández (SLP), Culiacán (Sin.), Hermosillo (Son.), Huimanguillo (Tab.) y Oxkutzcab (Yuc.). Como punto de destino se consideró la capital de cada uno de los 32 estados. Para la matriz de distancias de zonas productoras a las distintas industrias productoras de jugo se tomaron los mismos puntos de origen y como destino final la capital de los estados de Nuevo León, Puebla, San Luis Potosí, Tabasco, Tamaulipas, Veracruz y Yucatán. Para las importaciones se tomó como punto de internación a Tijuana y como destinos la capital de los 32 estados. Finalmente, para las exportaciones se tomaron como origen los 27 poblados descritos anteriormente y como punto de salida la ciudad de Nogales, Sonora.

\section{Procesamiento de la información}

Los datos del modelo se refieren al promedio de tres años; cualquier valor promedio de septiembre a agosto se refiere a los años 2014, 2015 y 2016. La solución del modelo en los diferentes escenarios se obtuvo con el procedimiento MINOS escrito en el lenguaje GAMS.

\section{RESULTADOS Y DISCUSIÓN}

En el periodo promedio 2014-2016, la producción nacional de naranja para consumo en fresco fue de 2724 miles de toneladas, y el $51.2 \%$ de la oferta nacional se obtuvo en febrero, marzo, abril y mayo (Cuadro 1). Debido a que no se consideró el almacenamiento del producto, el consumo de la naranja fue igual a la producción, la cual no es uniforme en las regiones productoras, ya que casi $70 \%$ de la producción se concentra en los estados de Veracruz y Tamaulipas. En ese mismo año la distribución del consumo fue la siguiente: 2724 miles de toneladas se consumieron en fresco, 1533 miles de toneladas fueron adquiridas por la industria, $10.4 \%$ de la producción se perdió en mermas, se importaron 27.7 miles de toneladas y 55 mil toneladas fueron exportadas en fresco.

Los resultados del modelo en sus diferentes escenarios se presentan en los Cuadros 2, 3, 4 y 5. Si toda la producción hubiera sido comercializada a los precios observados en el año de análisis, entonces el ingreso y el costo totales de producción hubieran ascendido, respectivamente, a 5422 y 4000 millones de pesos mexicanos; estas cifras hubieran determinado una ganancia para los productores de 1422 millones de pesos mexicanos a nivel nacional. Las cifras anteriores sólo consideran la producción de naranja que es consumida en fresco y fueron calculadas con el precio al mayoreo promedio mensual, de ahí la probabilidad de que aquellos productores que hubieran vendido su cosecha en los días de menor precio no hubieran obtenido ganancias.

De acuerdo con los resultados obtenidos en los tres escenarios planteados, se puede apreciar que una política viable sería la planeación de la producción en el tiempo, ya que si la disponibilidad por región productora fuera igual en cada mes en que biológicamente se puede obtener la producción, entonces desaparecerían los excesos de oferta temporales. La política consistiría en tratar de uniformizar y mover la producción; por ejemplo, disminuir el volumen en el mes de marzo, que presenta mayor producción, y aumentarla en los meses contiguos al mismo, los cuales presentan menor producción (enero, febrero y abril). Esto 
Cuadro 1. Producción, consumo y comercio de naranja en México. Miles de toneladas.

\begin{tabular}{|c|c|c|c|c|c|c|}
\hline \multirow{2}{*}{ Mes } & \multirow{2}{*}{ Producción } & \multirow{2}{*}{ Importaciones } & \multicolumn{2}{|c|}{ Consumo } & \multirow{2}{*}{ Mermas } & \multirow{2}{*}{ Exportaciones } \\
\hline & & & Fresco & Industrial & & \\
\hline Septiembre & 90.0 & 1.8 & 50.3 & 31.7 & 9.4 & 0.4 \\
\hline Octubre & 363.3 & 0.9 & 191.6 & 133.4 & 37.8 & 1.4 \\
\hline Noviembre & 539.7 & 1.1 & 306.4 & 173.4 & 56.1 & 4.8 \\
\hline Diciembre & 359.3 & 1.9 & 207.4 & 109.9 & 37.4 & 6.6 \\
\hline Enero & 408.2 & 1.8 & 224.3 & 137.6 & 42.5 & 5.6 \\
\hline Febrero & 556.2 & 2.3 & 309.1 & 184.0 & 57.8 & 7.5 \\
\hline Marzo & 862.8 & 2.7 & 470.7 & 297.4 & 89.7 & 7.7 \\
\hline Abril & 558.8 & 3.0 & 322.9 & 172.4 & 58.1 & 8.3 \\
\hline Mayo & 468.3 & 2.6 & 276.2 & 138.4 & 48.7 & 7.5 \\
\hline Junio & 332.1 & 3.0 & 208.5 & 87.5 & 34.5 & 4.6 \\
\hline Julio & 116.7 & 3.3 & 74.6 & 32.8 & 12.1 & 0.5 \\
\hline Agosto & 126.7 & 3.3 & 82.0 & 34.8 & 13.2 & 0.1 \\
\hline Anual & 4782.1 & 27.7 & 2724.0 & 1533.3 & 497.3 & 55.0 \\
\hline
\end{tabular}

Fuente: Elaboración propia con datos de la salida del modelo.

Cuadro 2. Producción, almacenamiento y ganancia del productor de naranja por mes. Situación observada en 2014-2016.

\begin{tabular}{|c|c|c|c|c|c|c|}
\hline \multirow[t]{2}{*}{ Mes } & \multirow[t]{2}{*}{ Producción $^{\dagger}(\mathrm{t})$} & $\begin{array}{c}\text { Ingreso del } \\
\text { productor }\end{array}$ & $\begin{array}{l}\text { Costo de } \\
\text { producción }\end{array}$ & $\begin{array}{l}\text { Costo de } \\
\text { almacén }\end{array}$ & $\begin{array}{l}\text { Ganancia } \\
\text { productor }\end{array}$ & $\begin{array}{c}\text { Ganancia } \\
\text { intermediario }\end{array}$ \\
\hline & & \multicolumn{5}{|c|}{ Millones de pesos mexicanos } \\
\hline Septiembre & 50,308 & 94 & 68 & 0 & 26 & 156 \\
\hline Octubre & 191,620 & 389 & 274 & 0 & 115 & 495 \\
\hline Noviembre & 306,442 & 706 & 454 & 0 & 252 & 626 \\
\hline Diciembre & 207,394 & 538 & 302 & 0 & 236 & 435 \\
\hline Enero & 224,286 & 487 & 320 & 0 & 167 & 547 \\
\hline Febrero & 309,124 & 697 & 445 & 0 & 252 & 640 \\
\hline Marzo & 470,693 & 837 & 690 & 0 & 147 & 904 \\
\hline Abril & 322,856 & 595 & 500 & 0 & 95 & 774 \\
\hline Mayo & 276,248 & 438 & 425 & 0 & 13 & 760 \\
\hline Junio & 208,476 & 353 & 297 & 0 & 56 & 595 \\
\hline Julio & 74,593 & 146 & 109 & 0 & 37 & 217 \\
\hline Agosto & 81,997 & 142 & 116 & 0 & 26 & 265 \\
\hline Anual & $2,724,037$ & 5422 & 4000 & 0 & 1422 & 6414 \\
\hline
\end{tabular}

†Sólo se consideró la producción que es consumida en fresco. Fuente: Elaboración propia con datos de la salida del modelo.

sería una medida que podría lograrse a través de podas del naranjo (Padrón y Rocha, 2017) o a través de la aplicación de ácido giberélico $\left(\mathrm{AG}_{3}\right)$ y ethefon para retrasar y adelantar la producción, respectivamente (Soto et al., 1994).

Esta medida genera efectos positivos sobre la ganancia del productor, los cuales se pueden observar en los Cuadros 2 y 4; por efecto de una mejor planeación de la producción, la ganancia del productor incrementaría en 32 millones de pesos (de 1422 a 1454 millones de pesos). El hecho de que 33 \% de la producción de naranja a nivel nacional se obtenga en condiciones de riego hace posible instrumentar un calendario de riegos para lograr el objetivo de la política. En términos del efecto sobre la ganancia, se puede observar que la planeación de la producción en el tiempo es la mejor opción; sin embargo, Espinoza 
Cuadro 3. Producción, almacenamiento y ganancia del productor de naranja por mes. Escenario con almacenamiento en meses de mayores excesos de oferta.

\begin{tabular}{lcccccc}
\hline \multirow{2}{*}{ Mes } & Producción ${ }^{\dagger}(\mathrm{t})$ & $\begin{array}{c}\text { Ingreso del } \\
\text { productor }\end{array}$ & $\begin{array}{c}\text { Costo de } \\
\text { producción }\end{array}$ & $\begin{array}{c}\text { Costo de } \\
\text { almacén }\end{array}$ & $\begin{array}{c}\text { Ganancia } \\
\text { productor }\end{array}$ & $\begin{array}{c}\text { Ganancia } \\
\text { intermediario }\end{array}$ \\
\cline { 3 - 7 } Septiembre & 50,306 & 95 & 69 & 0 & 26 & 155 \\
Octubre & 191,619 & 395 & 274 & 0 & 121 & 490 \\
Noviembre & 306,441 & 725 & 451 & 0 & 274 & 606 \\
Diciembre & 207,395 & 533 & 300 & 0 & 233 & 447 \\
Enero & 224,286 & 486 & 320 & 0 & 166 & 548 \\
Febrero & 309,125 & 698 & 446 & 0 & 252 & 645 \\
Marzo & 470,694 & 625 & 429 & 14 & 182 & 652 \\
Abril & 322,855 & 513 & 437 & 16 & 60 & 758 \\
Mayo & 276,247 & 452 & 450 & 16 & -14 & 807 \\
Junio & 208,478 & 318 & 276 & 17 & 25 & 579 \\
Julio & 74,593 & 263 & 274 & 10 & -21 & 635 \\
Agosto & 81,998 & 306 & 266 & 3 & 37 & 605 \\
Anual & $2,724,037$ & 5409 & 3992 & 76 & 1341 & 6927 \\
\hline
\end{tabular}

'Sólo se consideró la producción que es consumida en fresco. Fuente: Elaboración propia con datos de la salida del modelo.

Cuadro 4. Producción, almacenamiento y ganancia del productor de naranja por mes. Escenario en el cual la producción de marzo disminuye y aumenta la de enero, febrero y abril.

\begin{tabular}{|c|c|c|c|c|c|c|}
\hline \multirow[t]{2}{*}{ Mes } & \multirow[t]{2}{*}{ Producción $^{\dagger}(\mathrm{t})$} & $\begin{array}{l}\text { Ingreso del } \\
\text { productor }\end{array}$ & $\begin{array}{l}\text { Costo de } \\
\text { producción }\end{array}$ & $\begin{array}{l}\text { Costo de } \\
\text { almacén }\end{array}$ & $\begin{array}{l}\text { Ganancia } \\
\text { productor }\end{array}$ & $\begin{array}{c}\text { Ganancia } \\
\text { intermediario }\end{array}$ \\
\hline & & \multicolumn{5}{|c|}{ Millones de pesos mexicanos } \\
\hline Septiembre & 50,308 & 91 & 69 & 0 & 22 & 159 \\
\hline Octubre & 191,623 & 396 & 277 & 0 & 119 & 490 \\
\hline Noviembre & 306,442 & 708 & 445 & 0 & 263 & 624 \\
\hline Diciembre & 207,394 & 522 & 299 & 0 & 223 & 456 \\
\hline Enero & 224,789 & 494 & 320 & 0 & 174 & 543 \\
\hline Febrero & 357,251 & 782 & 512 & 0 & 270 & 703 \\
\hline Marzo & 332,671 & 678 & 488 & 0 & 190 & 739 \\
\hline Abril & 412,250 & 679 & 626 & 0 & 53 & 952 \\
\hline Mayo & 276,248 & 447 & 433 & 0 & 14 & 739 \\
\hline Junio & 208,476 & 363 & 301 & 0 & 62 & 602 \\
\hline Julio & 74,593 & 138 & 103 & 0 & 35 & 240 \\
\hline Agosto & 81,997 & 142 & 113 & 0 & 29 & 276 \\
\hline Anual & $2,724,042$ & 5440 & 3986 & 0 & 1454 & 6523 \\
\hline
\end{tabular}

'Sólo se consideró la producción que es consumida en fresco. Fuente: Elaboración propia con datos de la salida del modelo.

et al. (2005) señalaron la existencia de un bajo nivel de organización de los productores en el sector agrícola, quienes actúan de manera individual, lo que debilita su poder de negociación frente a los intermediarios; por tanto, si se supera el reto de organizar a los productores la medida podría implementarse.

El siguienteescenario analizado fueel dealmacenamiento de la fruta en los meses de mayor oferta para uniformizar el flujo de producto destinado al mercado, el cual demostró 
Cuadro 5. Producción, almacenamiento y ganancia del productor de naranja por mes. Escenario en el que la industria compra el $20 \%$ de la producción del mes 4 al 9.

\begin{tabular}{|c|c|c|c|c|c|c|}
\hline \multirow[t]{2}{*}{ Mes } & \multirow{2}{*}{ Producción $^{\dagger}(\mathrm{t})$} & $\begin{array}{l}\text { Ingreso del } \\
\text { productor }\end{array}$ & $\begin{array}{l}\text { Costo de } \\
\text { producción }\end{array}$ & $\begin{array}{l}\text { Costo de } \\
\text { almacén }\end{array}$ & $\begin{array}{l}\text { Ganancia } \\
\text { productor }\end{array}$ & $\begin{array}{c}\text { Ganancia } \\
\text { intermediario }\end{array}$ \\
\hline & & \multicolumn{5}{|c|}{ Millones de pesos mexicanos } \\
\hline Septiembre & 50,308 & 96 & 69 & 0 & 27 & 154 \\
\hline Octubre & 191,623 & 394 & 275 & 0 & 119 & 489 \\
\hline Noviembre & 306,442 & 703 & 458 & 0 & 245 & 627 \\
\hline Diciembre & 185,419 & 475 & 270 & 0 & 205 & 410 \\
\hline Enero & 196,756 & 432 & 285 & 0 & 147 & 501 \\
\hline Febrero & 272,325 & 615 & 388 & 0 & 227 & 610 \\
\hline Marzo & 411,213 & 764 & 599 & 0 & 165 & 872 \\
\hline Abril & 288,366 & 497 & 437 & 0 & 60 & 775 \\
\hline Mayo & 248,567 & 394 & 381 & 0 & 13 & 741 \\
\hline Junio & 208,476 & 360 & 300 & 0 & 60 & 633 \\
\hline Julio & 74,593 & 128 & 105 & 0 & 23 & 253 \\
\hline Agosto & 81,997 & 132 & 116 & 0 & 16 & 290 \\
\hline Anual & $2,516,085$ & 4990 & 3683 & 0 & 1307 & 6355 \\
\hline
\end{tabular}

†Sólo se consideró la producción que es consumida en fresco. Fuente: Elaboración propia con datos de la salida del modelo.

no ser una opción factible para alcanzar el objetivo planteado, ya que en el Cuadro 3 se puede observar que las ganancias al productor se reducirían en 81 millones de pesos. Este resultado responde a los altos costos en que se incurre al almacenar productos perecederos, ya que éstos requieren de alta especialización técnica, como la atmósfera controlada, en los almacenes y bodegas, la cual requiere de sensores para compuestos orgánicos volátiles, transmisores y detectores, así como de refrigerantes. También se presentan otras limitantes, como el hecho de que la naranja no puede ser almacenada por un periodo mayor a tres meses para conservar su calidad (PérezAparicio et al., 2007), que el volumen almacenado mensual tendría que ser igual a la capacidad de los almacenes para no incurrir en subutilización de espacios y que la cantidad almacenada tendría que ser inferior a la producción mensual.

Por otro lado, se requiere una gran cantidad de recursos para construir la infraestructura de almacenamiento requerida para este proceso. Las mermas inherentes al proceso de almacenamiento serían otra limitante, ya que disminuirían el ingreso y la ganancia de los productores. Las mermas se presentan por escaldado a consecuencia de las bajas temperaturas, por la pudrición y por magulladuras del producto durante las maniobras durante el almacenamiento.

El tercer y último escenario consideró que la industria de la naranja compra el 20 \% de la producción en seis de los 
meses con mayor producción. Los resultados para este escenario indican que la ganancia del productor disminuiría en 115 millones de pesos mexicanos. Los ingresos del productor y los costos de producción se ubicarían en 4990 y 3683 millones de pesos mexicanos, de tal manera que se establecería un ingreso bruto por 1307 millones de pesos mexicanos; sin embargo, en este escenario la ganancia unitaria disminuiría de 522 a 519 pesos mexicanos por tonelada, lo que indica la no factibilidad de este escenario.

Se estimaron efectos negativos con la implementación de políticas de almacenamiento y compra por parte de la industria sobre la ganancia del productor; ésto es causado por el alto margen de comercialización del intermediario, que llega a representar hasta $61 \%$ del precio al mayoreo. Como se puede observar en el Cuadro 2 la ganancia del intermediario es hasta cinco veces mayor que la ganancia del productor. En la medida en que se logre la organización de los productores y el ordenamiento del mercado, entonces aumentará el poder de negociación del productor, permitiéndole obtener una mayor ganancia.

\section{CONCLUSIONES}

De las soluciones analizadas del modelo de programación lineal, la mejor opción para contribuir a la solución del problema de precios bajos en los meses de mayor producción de naranja y maximizar la ganancia del productor es la planeación de la producción en el tiempo; esto se podría lograr desfasando las cosechas a través de labores culturales y la aplicación de retardantes y estimulantes químicos a las plantaciones. Políticas como el almacenamiento para un control de flujos al mercado y el envío de cierta cantidad de la producción a las industrias en los meses de mayor producción tendrían un impacto negativo sobre el ingreso y ganancia del productor. La instrumentación de la política de almacenamiento se enfrentaría a obstáculos como la obtención de recursos financieros para la construcción de infraestructura de almacenes y la falta de la organización de los productores. Los intermediarios alcanzan altos niveles de ingresos en la cadena comercial de la naranja, superior en cinco veces al de los productores; por tanto, se recomienda que estos últimos busquen ordenar el mercado de naranja a través de la planeación de la producción en el tiempo. Adicionalmente, se debe fomentar la organización para lograr convenios directamente con los mercados mayoristas, que garanticen que el productor obtenga mayor margen de ganancia.

\section{BIBLIOGRAFÍA}

AMS/USDA, Agricultural Marketing Service/United States Department of Agriculture (2019) Commodities covered by marketing orders. Agricultural Marketing Service, United States Department of
Agriculture. Washington, D. C. https://www.ams.usda.gov/ rules-regulations/moa/commodities (February 2019)

De Barros J. R. M, A. L. M. de Barros e M. P. Cypriano (2016) O mercado da Citricultura no Brasil e as suas Novas Perspectivas. CitrusBr Associação Nacional dos Exportadores de Sucos Cítricos. São Paulo, Brasil. $63 \mathrm{p}$

Espinoza A. J. J., I. Orona C. y P. Cano R. (2005) Situación y tendencias en las actividades de producción y comercialización del melón (Cucumis melo L.) en la Comarca Lagunera, México. Agrofaz 5:801-811.

FIRA, Fideicomisos Instituidos en Relación con la Agricultura (2017) Sistema de costos agrícolas. Resumen de costos. Fideicomisos Instituidos en Relación con la Agricultura. Morelia, Michoacán. https://www.fira.gob.mx/Nd/Agrocostos.jsp (Diciembre 2018)

HLPE, Grupo de Alto Nivel de Expertos en Seguridad Alimentaria y Nutrición (2011) Volatilidad de los precios y seguridad alimentaria. Un informe del Grupo de Expertos de Alto Nivel sobre Seguridad Alimentaria y Nutrición del Comité de Seguridad Alimentaria Mundial. Organización de las Naciones Unidas para la Alimentación y la Agricultura. Roma. 98 p.

INIFAP, Instituto Nacional de Investigaciones Forestales, Agrícolas y Pecuarias (2018) Costos del paquete tecnológico de la naranja en Veracruz. Instituto Nacional de Investigaciones Forestales, Agrícolas y Pecuarias. Martínez de la Torre, Veracruz. 5 p.

Macías M. A. (2000) La importancia de las organizaciones de productores en la hortofruticultura de México. El caso de la sandía en la costa de Jalisco. Carta Económica Regional 13:3 14.

Padrón C. J. E. y M. A. Rocha P. (2007) La poda de los cítricos. Folleto Técnico No. 7. Campo Experimental General Terán, INIFAP General Terán, Nuevo León, México. 46 p.

Pérez-Aparicio J., L. Zapata-Soberá, V. Lafuente-Rosales y M. Á. ToledanoMedina (2007) Almacenamiento de naranjas CV. "Salustiana" y cv. "Valencia" y su influencia en la calidad del fruto (I). Revista Iberoamericana de Tecnología Postcosecha 8:66-73.

Powers N. J. (1990) Federal Marketing Orders for Fruits, Vegetables, Nuts, and Specialty Crops. Agricultural Economic Report 629 Economic Research Service, United States Department of Agriculture. Washington, D. C. 34 p.

Ramos-Sandoval I. N., J. A. García-Salazar y M. Borja-Bravo (2018) Fluctuaciones estacionales y cíclicas de los precios del azúcar en México. Revista Fitotecnia Mexicana 41:317-325, https://doi. org/10.35196/rfm.2018.3.317-325

SCT, Secretaría de Comunicaciones y Transporte (2017) Traza tu ruta, punto a punto. Secretaría de Comunicaciones y Transportes. Ciudad de México. http://app.sct.gob.mx/sibuac_internet/ControllerUI ?action=cmdEscogeRuta (Diciembre 2017).

SIAP, Servicio de Información Agroalimentaria y Pesquera (2016) Avance de siembras y cosechas. Resumen por estado. Secretaría de Agricultura, Ganadería, Desarrollo Rural, Pesca y Alimentación. Ciudad de México. http://infosiap.siap.gob.mx:8080/agricola_ siap_gobmx/ResumenProducto.do (Julio de 2018)

SIAP, Servicio de Información Agroalimentaria y Pesquera (2019) Consulta por fracción arancelaria. Secretaría de Agricultura, Ganadería, Desarrollo Rural, Pesca y Alimentación. Ciudad de México. https://w6.siap.gob.mx/comercio/con_fracciona.php (Enero 2019).

SIAP, Servicio de Información Agroalimentaria y Pesquera (2020) Anuario estadístico de la producción agrícola.. Secretaría de Agricultura y Desarrollo Rural. Ciudad de México. https://nube.siap.gob. $\mathrm{mx} /$ cierreagricola/ (Abril 2020).

SNIIM, Sistema Nacional de Información e Integración de Mercados (2016) Mercados Nacionales. Resumen semanal de precios al mayoreo de frutas y hortalizas. Sistema Nacional de Información e Integración de Mercados, Secretaría de Economía. Ciudad de México. http://www.economia-sniim.gob.mx/nuevo/ (Enero 2018).

Soto O. M., J. R. Espinoza E. y G. Almaguer V. (1994) Desfasamiento de cosecha en naranja (Citrus sinensis (L) Osbeck) cv Washington Navel en El Progreso, municipio de Tenango de Doria, Hgo Revista Chapingo Serie Horticultura 2:187-190.

USDA, United States Department of Agriculture (2017) Mexico: citrus annual. United States Department of Agriculture. Washington D. C., USA. https://www.fas.usda.gov/data/mexico-citrus- 
